\title{
$\mathbf{X}$.
}

\section{Zur Kenntniss der Wirkung der Buttersäure und $\beta$-0xybuttersäure.}

(Aus dem Pbysiologischen Institut in Berlin.)

Von Dr. Wilhelm Sternberg, prakt. Arzt in Berlin.

Den ersten Hinweis, dass es sich bei dem Symptomencomplex des Coma diabeticum, den bereits Prout und 1854 v. Dusch beschrieben und 20 Jahre darauf Kussmaul and Frerichs ${ }^{2}$ als ganz charakteristisches Krankheitsbild aufstellten, um abnorme Stoffwechsel-Vorgänge handele, hat Gerhardt ${ }^{3}$ im Jahre 1865 erbracht und zwar durch den Fund jener obstartig riechenden Substanz, die dem Harn auf EisenchloridZusatz die braunrothe Färbung verlieh, und welche or für Acetessigester hielt. Deichmüller ${ }^{4}$ und Tollens" wiesen bald nach, dass es sich um Acetessigsäure handele, weshalb $\mathrm{Jacksch}^{5}$ später vom Coma diaceticum sprach. In eine ganz neue Phase trat die Theorie der Autointoxication durch die Beobachtungen von Coranda ${ }^{6}$ und besonders Hallervorden ${ }^{7}$ im Jahre 1880, dass nehmlich im Harn von manchen Diabetikern eine ausserordentliche Steigerung des Ammoniaks statt hat. Gaethgens ${ }^{8}$ bestimmte nun sämmtliche bekannten Säuren und Basen im Harn und kam so auf Grund theoretischer Berechnungen zu dem Schluss, dass in dem stets sauer reagirenden Harn dieser Diabetiker mit vermehrter Ammoniak-Ausscheidang eine noch unbekannte Säure vorhanden sein müsse, welche Säure 3 Jahre darauf Stadelmann ${ }^{9}$ fand und bei der Analyse als $\alpha$-Crotonsäure bestimmte. Schon im nächsten Jahre gelang es $\mathrm{K} \ddot{\mathrm{u}} \mathrm{z}^{10}$ und ganz unabhängig von ihm auch Minkowski ${ }^{11}$, die Säure als $\beta$-Oxybuttersäure $\mathrm{CH}_{3} \cdot \mathrm{CH}(\mathrm{OH}) \cdot \mathrm{CH}_{2} \cdot \mathrm{COOH}$, als die Oxysäure der IV. Reihe darzustellen, die bei der Destillation leicht in $\alpha$-Crotọnsäure übergeht. Da, wie aus den Formeln er- 
sichtlich, diese neue Säure

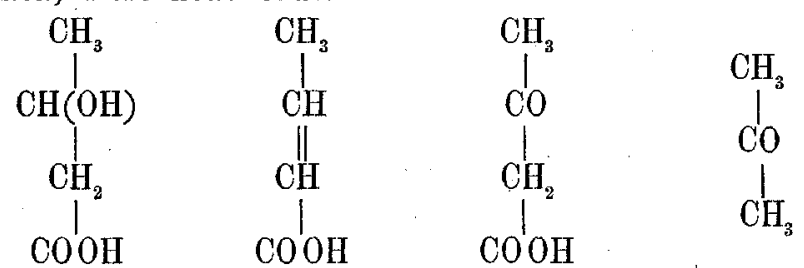

in naher Beziehung zu den früher gefundenen Substanzen steht, so erklärten sich einerseits die früheren Befunde ebenso leicht, wie das Erscheinen der $\beta$-Oxybuttersäure andererseits als Vorstufe dieser Substanzen bekräftigt erschien.

Seitdem steht die $\beta$-Oxybuttersäure im Vordergrunde des Interesses. Nachdem man mit Acetessigsäure, Aceton und den verwandten Körpern Verfütterungsversuche bei gesunden Thieren und bei Diabetikern und zwar mit negativem Erfolg gemacht hatte, lag es daher nahe, Versuche mit der neu entdeckten Oxysäure der IV. Reihe anzustellen, um zu sehen, ob ihr vielleicht eine specifisch toxische Wirkung zukomme, die das Coma diabeticum zu erklären im Stande wäre. Wenngleich dies a priori gar nicht, ja sogar eher das Gegentheil anzunehmen war, dass nehmlich diese Oxysäure nicht giftig sei, und zwar deshalb nicht, weil die $\beta$-Oxybuttersäure ${ }^{12}$, die sich im normalen Harn nie findet, vom Diabetiker Jahre lang ausgeschieden wird, so kann doch ein Zusammenhang zwischen dem Auftreten dieser Säure und dem Coma diabeticum nicht ganz von der Hand gewiesen werden, da erfahrungsgemäss das Coma im Verzuge ist, wo die Säure sich zeigt. Ein Umstand kam aber noch hinzu, welcher ganz besonders geeignet erschien, die Aufmerksamkeit auf die Oxysäure der IV. Reihe zu lenken. Hatte schon Walter ${ }^{13}$ durch seine Experimente dargethan, dass die Intoxication mit anorganischen Säuren eine grosse Aehnlichkeit mit dem Coma diabeticum bezüglich der eigenthümlichen Dyspnoe im Beginne, sowie der Steigerung der Respirations-Frequenz und der tiefen, mühsamen Athemzüge, und zwar hervorgebracht durch die Alkali-Entziehung, hat, so berichtete Binz ${ }^{14}$ im Jahre 1885 in der medicinischen Section der Niederrheinischen Gesellschaft für Naturheilkunde, ebenso im Jahre darauf auf dem Congress $^{15}$ für innere Medicin, dass die normale, nicht hydroxy- 
lirte Buttersäure, die Fettsäure der IV. Reihe, Erscheinungen macht, die an's Coma diabeticum erinnern. Andererseits war es bekannt, dass die Oxysäuren sich nicht wesentlich anders wie die nicht hydroxylirten Säuren verhalten, so die Oxybenzoesäure wie die Benzoesäure. Schon im Jahre 1864 hatte C. 0. Weber ${ }^{16}$ sehr auffallende Depressionen des Hirns bei Thieren gesehen, wenn er ihnen intravênös die freilich nicht neutralisirte, freie Buttersäure injicirte, Zustände, die ihn an die urämische Autointoxication erinnerten. Mayer's ${ }^{15}$. Untersuchungen führten zu dem Ergebniss, dass die Buttersäure zumal auf Katzen nach intravenöser Application derart wirkte, dass sie bei kleinen Dosen in einen Schlaf, bei grösseren Dosen in ein Coma verfallen, das bei geeigneten Dosen in den Tod übergeht. I. Munk ${ }^{17}$ beobachtet bei intravenöser Injection an Kaninchen, die er zwecks Studiums der Einwirkung der Buttersäure auf den Gaswechsel curarisirt hatte, dass der Herzschlag sofort kräftiger und häufiger wird, und die Darm-Peristaltik angeregt wurde.

Um mich zunächst von der Wirkung der Buttersäure zu überzeugen, wiederholte ich diese Versuche und stimmte deren Ergebniss mit Mayer's Resultaten überein; es zeigte sich nehmlich, dass gerade Katzen für Buttersäure empfindlich sind und besonders bei intravenöser Application in Schlaf verfallen.

\begin{tabular}{|c|c|c|c|c|c|c|c|}
\hline No. & Thierart & $\begin{array}{c}\text { Ge- } \\
\text { wicht } \\
\mathrm{g}\end{array}$ & $\mathbf{g}$ & pro $\mathrm{kg}$ & $\begin{array}{l}\text { Art der } \\
\text { Einver- } \\
\text { leibung }\end{array}$ & $\begin{array}{c}\text { Concentra- } \\
\text { tion des } \\
\text { Natrium- } \\
\text { salzes } \\
\text { pCt. }\end{array}$ & Wirkung \\
\hline $\begin{array}{ll}\text { 1. I. } & \text { I. } \\
\text { 2. II. }\end{array}$ & Kaninchen & $\begin{array}{r}1170 \\
981\end{array}$ & $\begin{array}{l}3,0 \\
1,2\end{array}$ & $\begin{array}{l}2,564 \\
1,21\end{array}$ & intravenös & $\begin{array}{l}20 \\
12\end{array}$ & $\begin{array}{c}\text { Tod } \\
\text { Wirkung }\end{array}$ \\
\hline $\begin{array}{ll}\text { 3. } & \text { I. } \\
\text { 4. } & \text { II. } \\
\text { 5. } & \text { III. } \\
6 . & \text { IV. } \\
\text { 7. } & \text { V. } \\
\text { 8. } & \text { VI. }\end{array}$ & $\begin{array}{c}\text { Katze } \\
- \\
- \\
- \\
- \\
-\end{array}$ & $\begin{array}{l}2200 \\
2550 \\
1350 \\
2550 \\
1250 \\
1300\end{array}$ & $\begin{array}{l}2,0 \\
5,0 \\
1,0 \\
3,0 \\
1,0 \\
3,0\end{array}$ & $\begin{array}{l}0,909 \\
1,960 \\
0,740 \\
1,176 \\
0,800 \\
2,307\end{array}$ & $\begin{array}{c}- \\
\text { subcutan } \\
- \\
\text { intravenös } \\
- \\
\text { subcutan }\end{array}$ & $\begin{array}{r}20 \\
20 \\
20 \\
26 \\
20 \\
6\end{array}$ & $\begin{array}{c}\text { Tod } \\
\text { keine Wirkung } \\
- \\
\text { Schlaf } \\
- \\
-\end{array}$ \\
\hline $\begin{array}{l}\text { 9. I. } \\
\text { 10. II. } \\
\text { 11. III. }\end{array}$ & $\begin{array}{c}\text { Froseh } \\
- \\
-\end{array}$ & $\begin{array}{l}50 \\
48 \\
50\end{array}$ & $\begin{array}{l}0,5 \\
0,2 \\
0,3\end{array}$ & E & $\begin{array}{l}- \\
- \\
-\end{array}$ & $\begin{array}{l}10 \\
10 \\
10\end{array}$ & $\begin{array}{c}\text { sebr starke Wirkung } \\
\text { schwache } \\
\text { Wirkung }\end{array}$ \\
\hline
\end{tabular}

Der erste Frosch, der $0,1 \mathrm{~g}$ buttersaures Natrium erhalten hat, zeigt nur die Erscheinung, dass er die weggespreizten Beine Archiv f. pathol. Anat. Bd. 152. Hft. 2 . 
langsam und träge wieder anzieht. Nach der zweiten Dosis von $0,1 \mathrm{~g}$ lässt er sich zwar leicht auf den Rücken legen, erhebt sich aber nach kurzer Zeit wieder und erholt sich bald.

Ein anderer Frosch, der $0,3 \mathrm{~g}$ buttersaures Natrium erhalten hat, lässt sich schon nach einigen Minuten ohne jedẹn Widerstand auf den Rücken legen und bleibt 6 Stunden ohne jede spontane Bewegung auf dem Rücken ruhig liegen, scheint also betäubt zu sein; nach weiteren 6 Stunden erholt er sich allmählich.

Der dritte Frosch, der $0,5 \mathrm{~g}$ buttersaures Natrium erhalten hat, lässt sich schon nach einer. Minute auf den Rücken legen und bleibt so 2 Tage lang liegen. Am dritten Tage wird er todt gefunden.

Ueber die Wirkung der Buttersäure, welche in der $\beta$-Stellung hydroxylirt wird, existiren in der Literatur nur wenige Angaben. Die ersten Versuche stellte Albertoni ${ }^{18}$ mit der $\beta$-Oxybuttersäure im Jahre ihrer Entdeckung an. Er sagte: "Ich stellte einige Versuche an, um zu sehen, ob die $\beta$-Oxybuttersäure im Organismus Acetessigsäure liefere und auf denselben irgend einen speciellen Einfluss ausübe. Ich stellte die $\beta$-Oxybuttersäure nach der Methode von Wislicenus dar. und brachte sie Thieren bei; doch fand ich im Harn derselben keine Acetessigsäure vor und beobachtete ich auch keine besonderen Wirkungen." Minkowski ${ }^{19}$ stellte einen Versuch beim Hunde an, dem er "Oxybuttersäure in der von Mayer $^{20}$ angewandten Dosis von $1 \mathrm{~g}$ subcutan injicirte, welcher absolut keine Vergiftungserscheinungen zeigte". Die ersten Verfütterungsversuche mit dem Natriumsalze der synthetisch nach der Methode von Wislicenus dargestellten $\beta$-Oxybuttersäure stellte Araki ${ }^{21}$ an, indém er Kaninchen, Hunden und Fröschen das Salz subcutan injicirte; und zwar vergiftete or die Thiere, um dem vermeintlichen Zersetzungsprodulte der Oxybuttersäure, dem Aceton, bezw. der Acetessigsäure za begegnen, sofort mit Kohlenoxydgas. Er findet fast immer Aceton und nimmt daher an, dass bei der Oxydation der $\beta$-Oxybuttersäure im Organismus Acetessigsäure als Zwischenstufe eintritt, während $W$ eintraud ${ }^{22}$ nach Einverleibung der Oxybuttersäure kèine Steigerung der Aceton-Ausscheidung constatiren konnte. Diese Versuche sind hinsichtlich der Wieder- 
gabe und ihrer Ausführung nicht ganz beweisend für die Giftigkeit, bezw. Ungiftigkeit der $\beta$-Oxybuttersäure; besonders gilt dies von Minkowski's ${ }^{19}$ Versuch. Abgesehen davon, dass die Dosis, die er angiebt von Mayer ${ }^{20}$ angewandt zu wissen, gar nicht die von Mayer bestimmte ist, hat auch Mayer bei der nicht hydroxylirten Buttersäure gerade jegliche Einwirkung vermisst, wenn sie subcutan verabfolgt wurde. Um also eine specifisch toxische Wirkung der $\beta$-Oxybuttersäure auszuschliessen, wie es die klinische Medicin heute thut, schienen mir diese Versuche nicht hinzureichen, weshalb ich einige Versuche mit dieșer Säure anstellte. Ausgehend von Mayer's und meinen eigenen Versuchen, aus denen ersichtlich war, dass gerade Katzen, wie dies auch schon Weber beobachtet hat, gegen Buttersäure so empfindlich sind und zwar besonders bei intravenöser Appliçation, hielt ich es für pöthig, zu diesen Versuchen auch intravenöse Injectionen und zu Versuchsthieren auch gerade Katzen zu wählen; zumal da, wie mehrfach angegeben wird, Katzen so leicht diabetisch werden, dass eine einfache Fesselung oder die Inanition allein genügt, ebenso wie Hunde, die wie Eber $^{23}$ nachgewiesen, einen wahren Diabetes mellitus mit Katarakt-Bildung und Coma diabeticum mit Auftreten der $\beta-0 x y-$ buttersäure gar nicht so selten haben.

\begin{tabular}{|c|c|c|c|c|c|c|c|}
\hline No. & Thierart & $\begin{array}{c}\mathrm{Ge}- \\
\text { wicht } \\
\mathrm{g}\end{array}$ & $\begin{array}{c}\text { Dosis } \\
\beta . \mathrm{O}_{4} \mathrm{H}_{7} \mathrm{O}_{3} \\
\mathrm{~g}\end{array}$ & $\begin{array}{c}\mathrm{Na} \\
\mathrm{pro} \mathrm{kg} \\
\mathrm{g}\end{array}$ & $\begin{array}{l}\text { Con- } \\
\text { cen- } \\
\text { tration } \\
\text { pCt. }\end{array}$ & $\begin{array}{l}\text { Verab- } \\
\text { folgung }\end{array}$ & Wirkung \\
\hline $\begin{array}{l}\text { 12. I. } \\
\text { 13. II. } \\
\text { 14. III. }\end{array}$ & $\begin{array}{c}\text { Katze } \\
- \\
-\end{array}$ & $\begin{array}{l}2650 \\
1070 \\
1000\end{array}$ & $\begin{array}{l}2,650 \\
2,033 \\
3,0\end{array}$ & $\begin{array}{l}1,0 \\
1,9 \\
3,0\end{array}$ & $\begin{array}{l}24,2 \\
24,2 \\
24,2\end{array}$ & intravenôs & $\begin{array}{c}\text { kein } \theta \\
-\end{array}$ \\
\hline $\begin{array}{l}\text { 15. I. I. } \\
\text { 16. II. }\end{array}$ & Erosch & $\begin{array}{l}70 \\
50\end{array}$ & $\begin{array}{c}1,21 \\
\text { Von d.Lösung: } \\
0,75\end{array}$ & $\begin{array}{l}17,3 \\
0,1815\end{array}$ & $\begin{array}{l}24,2 \\
24,2\end{array}$ & $\underbrace{\text { subcutan }}_{-}$ & $\begin{array}{c}\text { Tod in } 2 \text { Stunden } \\
\text { keine }\end{array}$ \\
\hline 17. III. & - & 45 & $\begin{array}{c}\text { Von d.Lösung: } \\
2 \mathrm{cem}\end{array}$ & 0,484 & 24,2 & - & Wirkung \\
\hline 18. IV. & - & 45 & $\begin{array}{c}\text { Pon d.Lösung: } \\
3 \mathrm{cem}\end{array}$ & 0,726 & 24,2 & - & - \\
\hline 19. V. & - & 45 & $\left|\begin{array}{c}\text { Von d.Lösung: } \\
1 \mathrm{cem}\end{array}\right|$ & 0,242 & 24,2 & - & - \\
\hline
\end{tabular}

3 Katzen im Gewichte von $1000-2650 \mathrm{~g}$ erhielten 1,0 bis $3,0 \mathrm{~g}$ des $\beta$-oxybuttersauren Natriums in einer 24,2 procentigen 
Concentration in die V. jugularis injicirt. Stets war eine Verstärkung der Herzaction zu bemerken, gemessen an der Intensität des. Herzstosses, wie eine solche auch das buttersaure Natrium bewirkt. In keinem Falle trat irgend welche Schlaffheit oder Mattigkeit auf.

Um mich zu überzengen, ob die eingegebene Dosis dẹ $\beta$-Oxybuttersäure der Verbrènnung anbeimgefallen ist oder nicht, untersuchte ich den Harn und zwar verfuhr ich folgendermaassen. Zur vorläufigen Orientirung, ob überhaupt merkliche Spuren von $\beta$-Oxybuttersäure in den Harn übergegangen sind, filtrirte ich den Harn und destillirte, nachdem ich zum Filtrat Phosphorsäure zugesetzt hatte. Wenn die $\beta$-Oxybuttersäure als solche, da sie ja selbst nicht flüchtig, ist, auch nicht aufzufinden war, so musste ich doch auf diese Weise ihrem Spaltungsprodukt, der $\alpha$-Crotonsäure begegnen, Stets war aber das Destillat so wenig sauer, wie vom Harn normaler Weise, so dass nur minimale Mengen einer $\frac{1}{10}$ N-Natronlauge zur Sättigung des Destillats genügten. Mithin waren nicht mehr als normaler Weise flüchtige Säuren im Harn, so dass zu schliessen war, dass die $\beta$-Oxybuttersäure der Verbrennung anheimgefallen ist.

Von den Fröschen im Gewichte von $45-70 \mathrm{~g}$ erhielten 4 eine Dosis von $0,75-5,0$ ccm derselben 24,2 procentigen Lösung der neutralisirten $\beta$-Oxybuttersäure in steigender Menge. Die geringste Dosis verursachte gar keine Wirkung, die nächst höhere Dosis aber Schlaffheit und Mattigkeit, die in vollständige Betäubung überging. Die höchste Dosis führte schon vach 2 Stunden zam Tode.

Diese Versuche ergeben also, was man a priori nicht erwarten sollte, dass die Buttersäure durch die Hydroxylirung, ausgenommen bei Fröschen, entgiftet ist.

Es lag mir nunmehr daran, die $\beta$-Oxybuttersäure einem diabetischen Thiere zu verabfolgen, um zu sehen, ob vielleicht die $\beta$-Oxybuttersäure auf den diabetischen Organismus anders einwirke. Zu diesem Behufe erzeugte ich einen Phlorhizin-Diabetes.

Eine Katze von $1500 \mathrm{~g}$ erhält $0,12 \mathrm{~g}$ Phlorhizin subcutan. Der in 24 Stunden gesammelte Harn wird auf das 20 fache verdünnt und giebt noch starke Reduction. Am nächsten Tage erhält dieselbe Katze $0,06 \mathrm{~g}$ Phlorhizin in die $\mathrm{V}$. jugularis inji- 
cirt, darauf $3 \mathrm{~g}$ oxybuttersaures Natrium. Das Thier ist munter und unterseheidet sich in nichts von einem normalen Thier. Im Harn jst massenhaft Traubenzucker nachweisbar. Es wird mit Phosphorsäure angesäuert und überdestillirt, und in dem Destillat ist nur so wenig flüchtige Säure, dass sie schon durch $1 \mathrm{ccm}$ einer $\frac{1}{10}$ Normallauge gesättigt wird, daher anzunehmen ist, dass die Oxybuttersäure verbrannt ist.

Nach den Verfütterungs - Versuchen an Thieren fragte es sich, wie die $\beta$-Oxybuttersäure auf den menschlichen Organismus wirkt, und zwar wie sie auf den gesunden Menschen, und wie sie auf den Diabetiker wirkt.

Die Gelegenheit zum Studium der Einwirkung der $\beta-0 x y-$ buttersäure auf den gesunden Menschen bot mir eine sonst körperlich gesunde, neurasthenische Frau, die ich längere Zeit wegen einer Venenthrombose in Behandlung habe. Ich gab derselben 5 Tage hindurch $5 \mathrm{~g} \beta$-Oxybuttersäure per os in Wasser gelöst. Die Nahrungs-Aufnahme war nahezu die gleiche. Die Methode, deren ich mich zum Nachweise der $\beta$-Oxybuttersäure im Harne bediente, war folgende: Da der Harn ganz minimale Spuren von Zucker enthielt, so wurden diese geringen Mengen von Traubenzucker zuerst vergohren, der filtrirte Harn zum dicken Syrup verdampft und mit gleichem Volumen concentrirter Schwefelsäure destillirt. Beim Abkühlen der Vorlage konnten Krystalle von der Crotonsäure nicht hergestellt werden. Zum Nachweis der Milchsäure bediente ich mich des Schütz'schen ${ }^{24}$ Verfahrens. Später verfuhr ich folgendermaassen: der mit Bleiessig versetzte Harn wird bis auf $\frac{1}{3}$ seines Volumens eingedampft, filtrirt und mit Schwefelwasserstoff gesättigt. Das bis zur Syrupdicke eingedampfte Filtrat wird im Scheidetrichter mit wasserfreiem Aether mehrmals ausgeschüttelt, letzterer verdunstet und der Rückstand mit Eisenchlorid auf Milchsäure geprüft.

Die nach diesen Methoden ausgeführten Untersuchungen ergaben, dass die Oxybuttersäure nicht ausgeschieden wurde, die Oxydationsprodukte ihrer optisch activen Isomeren, das Aceton und die Acetessigsäure so gut wie gar nicht im Harn aufzufinden waren; mithin ist die $\beta$-Oxybuttersäure verbrannt worden. Das Allgemeinbefinden oder gar das Sensorium war. niemals, in keiner Weise gestört, 


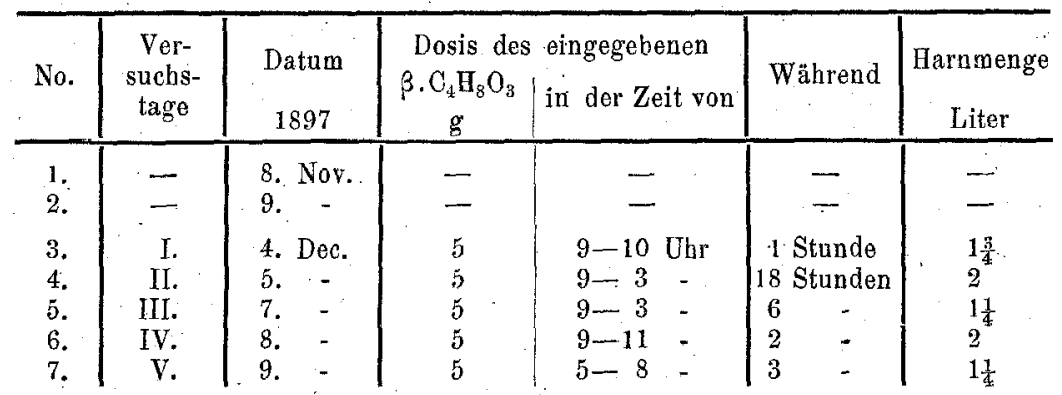

Aceton fand sich fast in jedem Harn in Spuren, Acetessigsäure, $\beta$-Oxybuttersäure

Nunmehr kam es mir darauf an, zu sehen, ob die $\beta-0 x y$ buttersäure auf den Diabetiker eine Wirkung ausübt. Zu diesem Zwecke wählte ich einen Casus gravis, einen Diabetiker, der schon über 10 Jahre krank ist und dessen Harn die Gerhardt'sche Probe giebt.

Herr M., geb. 1842, stammt aus ganz gesunder Familie. Im Jahre 1875 erhielt er einen schweren Schlag auf den Hinterkopf, seit 1884 ist er zuckerkrank und zwar schwankt der Zucker-Gehalt in den 13 Jahren seiner Krankheit zwischen 5 und 10pCt. Herr M. erhält an 3 Tagen je $5 \mathrm{~g}$ und 2 mal je $10 \mathrm{~g}$. $\beta$-Oxybuttersäure per os. Das Allgemeinbefinden ist nicht im mindesten gestört, das Sensorium in keiner Weise verändert. Im Destillat des mit Phosphorsäure angesäuerten Harnes war

\begin{tabular}{|c|c|c|c|c|c|}
\hline $\begin{array}{c}\text { Datum } \\
1897\end{array}$ & $\begin{array}{c}\text { Versuchs- } \\
\text { tage }\end{array}$ & $\begin{array}{c}\text { Dosis } \\
\text { der ein- } \\
\text { gegebenen } \\
\beta . \mathrm{C}_{4} \mathrm{H}_{8} \mathrm{O}_{3} \\
\mathrm{~g}\end{array}$ & Urinmenge & Reaction & $\begin{array}{l}\text { Spec. } \\
\text { Gew. }\end{array}$ \\
\hline 7. December & 一 & 一 & $2 \frac{1}{2}$ Liter & sauer & 1040 \\
\hline 8. - - & - & - & $2 \frac{1}{2} \quad-$ & $\cdot-$ & 1041 \\
\hline 9. - & - & - & $2 \frac{1}{4} \quad-$ & stark sauer & 1037,5 \\
\hline 10. & - & 一 & $2^{4}-$ & $-\quad-$ & $1030^{\circ}$ \\
\hline 13. & - & - & $1 \frac{1}{2} \quad-$ & sauer & 1030 \\
\hline 15. & - & - & $1 \frac{3}{4} \quad-$ & - & 1033 \\
\hline 17. - & - & 一 & $2 \frac{1}{2} \quad-$ & 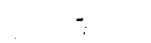 & 1035 \\
\hline 18. & I. & 10 & $1 \frac{3}{4}:-$ & sauer & 1033 \\
\hline 19. & II. & 5 & $1370 \mathrm{~g}$ & - & 1037 \\
\hline 20. & III. & 一 & $1 \frac{3}{4}$ Liter & - & 1034. \\
\hline 22. & IV. & 10 & $2^{7}=$ & $=$ & 1030 \\
\hline 23 & - & - & $2 \frac{1}{4}:$ & $:$ & 1034 \\
\hline
\end{tabular}




\begin{tabular}{|c|c|c|c|c|c|}
\hline Spec. Gew. & $\begin{array}{l}\text { Feste Be- } \\
\text { standtheile } \\
\text { in } 1 \text {. Liter }\end{array}$ & Reaction & $\begin{array}{c}\overline{\mathrm{U}} \\
\text { p Ct. }\end{array}$ & $\begin{array}{c}\stackrel{+}{\mathrm{U}} \\
\text { pCt. }\end{array}$ & Indican \\
\hline $\begin{array}{l}1,0195 \\
1,0183\end{array}$ & $\begin{array}{l}45,43 \\
43,74\end{array}$ & sauter & $\begin{array}{l}0,045 \\
0,04\end{array}$ & $\begin{array}{l}2,07 \\
1,77\end{array}$ & reichlich \\
\hline $\begin{array}{l}1,0227 \\
1,0197 \\
1,0263 \\
1,0260 \\
1,0267\end{array}$ & $\begin{array}{l}57,88 \\
50,23 \\
61,28 \\
60,58 \\
68,08\end{array}$ & $\begin{array}{c}- \\
- \\
- \\
- \\
\text { schwach sauer }\end{array}$ & $\begin{array}{l}- \\
- \\
0,057 \\
0,065 \\
0,055\end{array}$ & $\begin{array}{l}- \\
-\overline{4} \\
2,43 \\
3,722 \\
3,22\end{array}$ & $\begin{array}{c}\text { sebr reichlich } \\
\text { wenig } \\
\text { reichlich } \\
- \\
\text { sehr reichlicb }\end{array}$ \\
\hline
\end{tabular}

Milchsäure njemals. - Die Ausathmungsluft roch nie obstartig.

kaum eine Spur einer flüchtigen Säure nachweisbar, andererseits war auch der Ammoniakgehalt des Harns nicht vermehrt. Da zudem die Oxydationsprodukte der Oxybuttersäure nicht vermehrt waren, ist die Annahme berechtigt, dass der Diabetiker die Oxybuttersäure vollständig verbrannt hat.

All' diese Versuche haben also ergeben, dass die $\beta-0 x y-$ buttersäure die Giftigkeit. mit der Buttersäure nicht theilt. Freilich haben wir in unseren Experimenten stets mit der optisch inactiven, synthetisch dargestellten $\beta$-Oxybuttersäure operirt, während der Diabetiker die optisch-active Säure zur Ausscheidung bringt. Zwischen beiden durch ihr optisches Verhalten verschiedenen Isomeren besteht ja, wie man jetzt weiss, kein directer Gegensatz, ebenso wenig bei der Oxysäure der

\begin{tabular}{|c|c|c|c|c|}
\hline $\begin{array}{c}\text { Zucker- } \\
\text { gehalt } \\
\text { pCt. }\end{array}$ & $\begin{array}{l}\text { Acetessig- } \\
\text { säure }\end{array}$ & Aceton & $\begin{array}{r}\mathrm{NH}_{3} \\
\text { pCt.-g }\end{array}$ & Allgemeinbefinden \\
\hline $\begin{array}{l}8,0 \\
8,76 \\
6 \\
5 \frac{1}{2} \\
4 \\
4,6 \\
5,3\end{array}$ & $\begin{array}{c}\text { reichlich } \\
- \\
- \\
\text { vorhanden } \\
\text { reichlicb } \\
- \\
-\end{array}$ & $\begin{array}{c}\text { reichlich } \\
- \\
- \\
- \\
- \\
\text { vorhanden } \\
\text { reicblich }\end{array}$ & & \\
\hline $\begin{array}{l}5,4 \\
5,4 \\
5,4 \\
5,4 \\
5,4\end{array}$ & $\begin{array}{c}\text { vorbanden } \\
\text { reichlich }\end{array}$ & $\begin{array}{c}\text { vorhanden } \\
\text { reichlich } \\
\text { vorhanden } \\
-\end{array}$ & $\begin{array}{c}\overline{-} \\
0,0574 \\
0,0527 \\
0,0553\end{array}$ & nicht gestört \\
\hline
\end{tabular}


IV. Reihe, der Oxybuttersäure, wie bei der Oxysäure der III. Reihe, der Oxypropionsäure, der Milchsäure, welche beim Rheumatismus angeschuldigt wird, die Säuerung des Schweisses zu bedingen. Mit der optisch activen $\beta$-Oxybuttersäure hat Minkowski ${ }^{25}$ einen Versuch angestellt und zwar bei einem Hunde, der durch Pankreas-Exstirpation diabetisch gemacht war. Auch hier zeigte sich keine Wirkung. Es bleibt also thatsächlich für die Erklärung des Coma diabeticum nur die Annahme übrig, dass die Oxybuttersäure, wie es Minkowski ${ }^{19}$ und Kraus ${ }^{26}$ thatsächlich bei comatösen Diabetikern nachgewiesen haben, zu einer Alkali-Verarmung des Blutes führt, die ihrerseits das Coma bedingt.

Zum Schluss ist es mir eine angenehme Pflicht, Herrn Prof. I. Munk für die liebenswürdige Unterstützung meinen Dank auszusprechen.

$$
\text { I. i t e r a t u r. }
$$

1. Kussmaul, Znr Lehre vom Diabetes mellitus. Deutsches Archiv für klin. Med. Bd. 14. S. 1 ff. 1874.

2. Frerichs, Ueber plötzlichen. Tod und das Goma diabeticum. Zeitschr. für klin. Med. Bd. VI. S. 1.

3. Gerbardt, Wiener med. Presse. 28. 1865. Bd. VI. 1868.

4. Annalen der Chem. und Pharmac. Bd. 209. 22.

5. Jacksch, Ueber Acetonurie und Diaceturie. Berlin 1886.

6. Corenda, Ueber das Verhalten des Ammoniaks im menschlichen Organismus. Archiv für exp. Path. und Pharm. Bd. XII. 1880. Zeitschr. für physiol. Chem. Bd. VII. S. 487. 1883.

7. Hellervorden, Ueber die Ammoniak-Ausscheidung im Urin bei patbolog. Zuständen. Archiv für exp. Path. und Pharm. Bd. XII. S. 237 .

8. Gaethgens, Ueber Ammoniak-Ausscheidung. Zeitschr. für physiol. Chem. Bd. XIV.

9. Stadelmann, Ueber die Ursachen der patholog. Ammoniak-Ausscheidung beim Diabetes mellitus und Coma diabeticum. Archiv für exp. Path, und Pharm. Bd. XVI. 1883. S. 419.

10. Külz, Zeitschr. für Biologie. XX. 1884. - Arch. für exp. Patb. und Pharm: X VIII. 291.

11. Minkowski, Centralbl. fär die med. Wissensch. 1884. No. 15. Archiv für exp. Path. und Pharm. XVIII. 1884. - Zeitschr. für analyt. Chemie. 24. 153.

12. Derselbe, Arch. für exp. Path. und Pharm. Bd. XVIII. S. 44. 
13. Walter, Arch. für exp. Path. und Pharm. Bd. VII.

14. Binz, Congr. für innere Med. V. 175. 1886.

15. Mayer, Ueber die toxisehe Wirkung der niederen Fettsäuren. Archiv für exp. Path. und Pharm. XXI. 119. 1886.

16. C. 0 . Weber, Experimentelle Studien über Pyämie, Septicämie und Fieber: Deutsehe Klinik. 1864. S. 488.

17. Munk, Pflüger's Archiv. Bd. 46. 1890.

18. Albertoni, Ueber die Wirknng und Verwandlung einiger Stoffe im Organismus in Beziehung zur Pathogenese der Acetonämie und des Diabetes mellitus. Archị für exp. Path. und Pharm. XVIII. S. 238.1884 .

19. Minkow ski, Ueber Kohlensäuregehalt des Blutes bei Diabetes mellitus. Arbeiten aus der med. Königsberger Klinik. S. 175. 1888. und Archiv für experim. Path. XXXI. S. 182.

20. Mayer, Archiv für exp. Path. und Pharm. XXI.

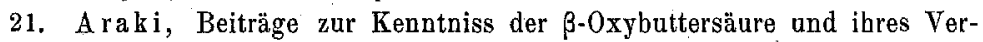
baltens im Organismus. Zeitschr. für physiol. Chemie. XVIII. 1894. S. 1.

22. Weintraud, Archiv für exp. Path. und Phàrm. XXXIV. 169.

23. Eber, Zwölf Fälle von Diabetes mellitus beim Hunde. Monatshefte für praktische Thierheilkunde. Bd. IX. 1897.

24. Zeitschr, für physiol. Chemie. 1894. XIX. 482.

25. Minkowski, Archiv für exp. Path. und Pbarm. XXXI. S.98. und XIX. S. 224.

26. Kraus, Zeitschr. für Heilkunde. Prag. Bd.X. S. 106. - Lépine, Revue de médecine. Tome VII. 1887. p. 224. 